\title{
Frühe digitale Poesie
}

\section{Christopher Strachey und Theo Lutz}

\author{
Toni Bernhart ${ }^{1,2} \cdot$ Sandra Richter ${ }^{1,2}$ \\ Angenommen: 12. Januar 2021 / Online publiziert: 9 . Februar 2021 \\ (c) Der/die Autor(en) 2021
}

\section{Zusammenfassung}

Die Idee, Poesie maschinell zu generieren, ist so alt wie die Maschinen selbst. Sie lässt sich seit dem Mittelalter beobachten und setzt sich fort bis in die Gegenwart, in der Storytelling-Experimente Algorithmen zur natürlichsprachigen Textgenerierung (NLG) nutzen. Die weltweit ersten Versuche, Poesie digital herzustellen, gelangen in den 1950er Jahren den Mathematikern Christopher Strachey (1916-1975) in Manchester und Theo Lutz (1932-2010) in Stuttgart. Durch zufallsmäßige Auswahl passfähiger Wörter und Sätze erzeugte Strachey 1952 auf einer Ferranti Mark I kurze Liebesbriefe. Dazu verwendete er das Programmierhandbuch von Alan Turing (1912-1954). Daneben schrieb Strachey Programme für das Dame-Spiel und zur computationellen Erzeugung von Musik. Lutz stellte 1959 mithilfe eines Programms im Freiburger Code auf einer Zuse Z 22 seine Stochastischen Texte her, wofür er Wortmaterial aus Franz Kafkas Roman Das Schloss (1926) verwendete und wobei er mit dem Philosophen Max Bense (1910-1990) und dem späteren Informatik-Pionier Rul Gunzenhäuser (1933-2018) kooperierte. Weil Lutz' Arbeitsunterlagen nahezu vollständig erhalten und im Deutschen Literaturarchiv Marbach für die Forschung zugänglich sind, gilt Lutz als ein literatur- und informatikgeschichtlich bedeutsamer Vertreter früher digitaler Poesie, die lange Zeit fast nur in avantgardistischen Zirkeln diskutiert wurde und kaum den Weg in eine breitere Öffentlichkeit fand. Erst aktuelle Debatten um sogenannte Künstliche Intelligenz (KI) lenken wieder die Aufmerksamkeit auf diese frühen Experimente, die ein faszinierender Mosaikstein der Technik-, Kultur- und Literaturgeschichte sind.

\section{Einleitung}

Die Idee, Poesie maschinell zu generieren, ist alt - vermutlich so alt wie die Maschinen selbst. Sie weist zurück auf die Kombinatorik: So versuchte bereits Ramon Lull (1232-1316) ein logisches Symbolsystem für alle Wissensbereiche zu entwickeln; sein Cant de Ramon spielt das kombinatorische Dichten in einem autobiografischen Gedicht durch. Die Nachahmungen und Erweiterungen des kombinatorischen Prinzips dauern Jahrhunderte an; sie reichen bis

Toni Bernhart

toni.bernhart@ilw.uni-stuttgart.de

Sandra Richter

sandra.richter@dla-marbach.de

1 Deutsches Literaturarchiv Marbach, Schillerhöhe 8-10, 71672 Marbach am Neckar, Deutschland

2 Institut für Literaturwissenschaft (NDL 1), Universität Stuttgart, Keplerstraße 17, 70174 Stuttgart, Deutschland zur Gruppe OULIPO (L'Ouvroir de Littérature Potenzielle), die seit 1960 besteht und seitdem wechselnde, oft prominente Besetzungen kennt. Auch Marcel Duchamp und Oskar Pastior waren Oulipolisten: Autoren, die ihre Texte aus einem Formzwang schrieben. Parallel zu und überlappend mit dieser Entwicklung findet man bereits in $\mathrm{Ge}$ org Philipp Harsdörffers Delitiae Mathematicae et Physicae (1651) einen ,Denckring“ mit 5 Scheiben, die gegeneinander verdreht 101.606.400 Wörter bilden [12, S. 517]. In Jonathan Swifts Gullivers Travels (1726) wiederum beeindrucken Zeichnungen von potenziellen Literaturmaschinen die Leser; sie werden mithilfe von Kurbeln in Bewegung gesetzt.

Die ersten Versuche digitaler, also mithilfe von Algorithmen und Computern erzeugter Poesie, knüpfen an diese Entwicklungen an. Schon die frühe digitale Literatur zeugt vom Entdeckergeist der Experimentatoren ebenso wie von ihrem literarischen Traditionswissen. Zugleich verdeutlicht diese Literatur, dass die Informatik - die damals noch nicht diesen Namen trug - in sehr viele Fachgebiete hineinragte. 
Denn es war nicht ausverhandelt, zu welchem Fach die Beschäftigung mit rechnenden Maschinen und Programmiersprachen gehören sollte. Zur Mathematik oder zur Elektrotechnik? Vielleicht auch zu Philosophie, Grammatik, Literatur oder Kunst? Der fächer- und spartenübergreifende Anspruch der Pioniere jedenfalls war groß - auf jeden Fall größer als das Interesse einzelner Fächer an den Möglichkeiten, die die Mensch-Maschine-Interaktion bieten konnte.

Frühe digitale Poesie hatte aber eine kurze Halbwertszeit. Zum einen wurde sie fast ausschließlich in kleinen avantgardistischen Zirkeln wahrgenommen und diskutiert, zum anderen waren die technischen Entwicklungen so rasant, dass künstlerische Experimente mit dem Computer oft schon nach kurzer Zeit als veraltet galten. Jahrzehntelang schien Hans Magnus Enzensbergers Poesie-Automat, 1974 entworfen und im Jahr 2000 für das Landsberger Lyrikfestival gebaut und danach prominent im Literaturmuseum der Moderne des Deutschen Literaturarchivs Marbach positioniert, wie ein großer Solitär. Erst die Netzliteratur, die im Zuge des breit verfügbaren Internets entstand und interaktive, partizipative und dialogische Ansätze vertrat, machte wieder auf frühe Versuche maschinengenerierter Poesie aufmerksam [22]. Ab Beginn des 21. Jahrhunderts bringen die Digital Humanities die traditionsreichen Geisteswissenschaften mit Informatik und Technikwissenschaften ins Gespräch. Sie rücken dabei auch historische Hard- und Software und deren Ergebnisse und Hervorbringungen in den Fokus medienarchäologischer und kulturwissenschaftlicher Forschung. Christopher Strachey in Manchester und Theo Lutz in Stuttgart waren in den 1950er-Jahren weltweit unter den ersten, denen die Generierung von Text mit einem programmierten Computer und mit literarischer oder ästhetischer Gestaltungsabsicht gelang.

\section{Christopher Strachey}

Christopher Strachey (1916-1975) studierte ab 1935 Mathematik am King's College der University of Cambridge, spielte in seiner Freizeit Klavier und lernte bald den 4 Jahre älteren Kommilitonen Alan Turing (1912-1954) kennen. Im Jahr 1938 erlitt Strachey einen psychischen Zusammenbruch, den sein Biograf Martin Campbell-Kelly auf eine persönliche Krise angesichts von Stracheys Homosexualität zurückführt [7, S. 21]. Dies legt nahe, im Anschluss etwa an Jacob Gaboury, der Strachey in seiner A Queer History of Computing (2013) behandelt [11], frühe digitale Poesie englischer Provenienz vielleicht im Rahmen einer erst noch zu konturierenden „Queer History of Digital Poetry“ $\mathrm{zu}$ erforschen. Nach Stracheys nicht besonders erfolgreichem Studienabschluss 1938 trat er eine Stelle als Physiker bei Standard Telephones and Cables Limited (STC) an. Hier arbeitete er unter anderem an geheimer militärischer For- schung zur Entwicklung von Röhren für zentrimetrisches Radar. Nach dem Krieg orientierte er sich beruflich neu und wurde Lehrer [7, S. 19-23].

Die Lektüre von Norbert Wieners Cybernetics or Control and Communication in the Animal and the Machine (1948) soll in Strachey die Begeisterung für Computer geweckt haben, schreibt Campbell-Kelly [7, S. 24]. Ende 1950 gelang es Strachey dank seiner Verbindungen zur Londoner Intellektuellenszene, einen Tag am National Physical Laboratory (NPL) in Teddington, Middlesex, zu verbringen. Im NPL wurde die Pilot ACE gebaut und das Laboratorium galt neben Cambridge und Manchester als eines der führenden britischen Zentren für Computertechnik. Nach seinem Besuch im NPL entwarf Strachey ein Programm für das Damespiel für die Pilot ACE. Als er im Frühjahr von der Ferranti Mark I erfuhr, die an der Manchester University installiert war, bat er Turing um ein Arbeitsexemplar des von diesem erarbeiteten Programmierhandbuchs für die Maschine, um sein Damespiel-Programm für die Mark I zu optimieren. Strachey führte Turing sein Programm vor und dieser war davon so beeindruckt, dass er ihn an die National Research and Development Corporation (NRDC) empfahl. Das NRDC war eine 1949 von der britischen Regierung gegründete Einrichtung, um wissenschaftliche Forschungsergebnisse, insbesondere aus der militärischen Forschung Großbritanniens während des Zweiten Weltkriegs, in industriell verwertbare Anwendungen und Patente zu überführen.

Das NRDC bot dem Quereinsteiger Strachey eine Stelle als Programmierer an, die er im Juni 1952 antrat. Er wirkte hier an unterschiedlichen Projekten mit, insbesondere an der Lösung logischer Probleme von Computern. Zweimal noch wechselte Strachey seinen Arbeitsort: Ab 1962 war er Forschungsassistent im Mathematical Laboratory der University of Cambridge und arbeitete an der Entwicklung der Combined Programming Language (CPL). Im Jahr 1965 ging er nach Oxford und wurde als freiberuflicher Berater mit dem Aufbau der Programming Research Group der Oxford University beauftragt. Mitten im Berufsleben verstarb Strachey 1975 plötzlich an einer infektiösen Hepatitis. Sein Nachlass liegt in der Bodleian Library [7, S. 24-25, S. 39].

\section{Love Letters}

Den spielerischen Charakter seiner Programmierarbeit trieb Strachey in mehrere Richtungen. Bereits 1951 entwickelte er zusammen mit Turing einen Algorithmus, der die Ferranti Mark I dazu brachte, „God Save the Queen“ zu spielen. Eine Azetatplatte mit einer von der BBC erstellten Tonaufzeichnung dieses Experiments wurde 2008 wieder aufgefunden und 2016 restauriert. Bei der Platte handelt sich um das weltweit älteste bekannte Beispiel einer Aufzeichnung von Computermusik [3, 8]. Weil es in den ersten Monaten 
seiner Arbeit an der NRDC im Sommer 1952 für Strachey nicht viel zu tun gab, verbrachte er die meiste Zeit mit der Perfektionierung seines Damespiel-Programms für die Mark I [7, S. 25]. In der Folge schließlich schrieb er ein Programm, das kleine Texte generieren konnte: Liebesbriefe nämlich. Auch wenn die Schilderung CampbellKellys etwas anekdotenhaft klingt, veranschaulicht sie doch deutlich, was in diesen Wochen geschah: „To keep himself amused he [Strachey] devised, with some aesthetic advice from his sister Barbara, a program that composed love letters by randomly selecting suitable words and phrases" [7, S. 25]. Amüsement, ästhetischer Anspruch, zufallsmäßige Auswahl passfähiger Wörter und Sätze - und das Ganze maschinengemacht. Dies waren die Ingredienzen des Einfalls, mit dem Strachey Literaturgeschichte schreiben sollte. Er gilt weltweit als der erste, dem die Kompilation sinnhafter Texte mit einem Computer gelang. Bezeichnenderweise handelte es sich dabei um Prosatexte mit übersichtlicher Struktur.

Zwei der kurzen Liebesbriefe, die Strachey 1952 erzeugte, veröffentlichte er in seinem Essay „The ,Thinking “ Machine“ (1954) im Literaturmagazin Encounter [21]. Strachey leitet seinen Beitrag mit einer persönlichen Beobachtung ein: Personen, die erfahren, dass er sich beruflich mit elektronischen Rechenmaschinen beschäftigt, lassen sich in 2 Gruppen unterteilen. Die einen, die er als unsicher oder politisch engagiert beschreibt (,,insecure or politically-minded“), fragen, wie lange es dauert, bis Maschinen ihnen die Arbeit wegnehmen. Die anderen wollen wissen, ob Computer denken können. Sie fragen das, weil sie die Bestätigung suchen, dass kein Computer denken kann.

Diese Fragen, führt Strachey fort, seien falsch gestellt. Denn Computer könnten nichts außer sehr schnell eine lange Reihe von Anweisungen ausführen. Sinn und Zweck der Computer seien unablösbar an ein von Menschen geschriebenes Programm gebunden. Ein Computer ohne Programm sei wie eine Schreibmaschine ohne jemanden, der darauf tippt, oder ein Klavier, das niemand spielt. Die Frage, folgert Strachey, könne nicht lauten, ob ein Computer dieses oder jenes kann, sondern müsse richtig lauten, ob ein Mensch in der Lage ist, ein Programm zu schreiben, das den Computer veranlasst, dieses oder jenes zu tun [21, S. 25]. Um diesen Gedankengang zu veranschaulichen, bringt Strachey das Beispiel eines vermeintlich dichtenden Computers und gibt 2 seiner computergenerierten Liebesbriefe wieder.

\section{Darling Sweetheart}

You are my avid fellow feeling. My affection curiously clings to your passionate wish. My liking yearns for your heart. You are my wistful sympathy: my tender linking.

Yours beautifully

M.U.C. [21, S. 26].

\section{Honey Dear}

My sympathetic affection beautifully attracts your affectionate enthusiasm. You are my loving adoration: my breathless adoration. My fellow feeling breathlessly hopes for your dear eagerness. My lovesick adoration cherishes your avid ardour.

Yours wistfully

M.U.C. [21, S. 26].

Der Computer, erläutert Strachey, könne stundenlang solche Briefe schreiben, ohne sich zu wiederholen. Das Prinzip sei ausgesprochen einfach (,,childishly simple“): Sätze nach vorgegebenem Muster werden zufällig mit Wörtern aus einer vorgegebenen Liste gefüllt. Zwei Muster seien für die zu generierenden Texte gegeben:

$$
\begin{aligned}
& \text { My }+[\text { Adjektiv }]+[\text { Substantiv }]+[\text { Adverb }]+[\text { Verb }]+ \\
& \text { your }+[\text { Adjektiv }]+[\text { Substantiv }]
\end{aligned}
$$

und

You are my $+[$ Adjektiv $]+[$ Substantiv $]$.

Die Platzhalter für Verben, Substantive, Adjektive und Adverbien füllt Strachey aus dem Synonymwörterbuch Thesaurus of English Words and Phrases (1852), das der Arzt und Lexikograf Peter Mark Roget (1779-1869) erstellte. Ferner gelten die kombinatorischen Regeln, dass der Text mit einer Wortfolge aus einer speziellen Liste mit Anredeformen beginnt, dass darauf 5 Sätze folgen, wobei die Wahl der 2 möglichen Satzmuster zufällig ist, und dass nach den 5 Sätzen der Text mit einer Grußformel nach dem Muster

Yours $+[$ Adjektiv $]+$ M.U.C.

endet. Der springende Punkt an dieser Textherstellung, urteilt Strachey, seien nicht die eigentümliche Würze (,,peculiar flavour") der Briefe, sondern die offensichtliche Rohheit des Schemas und die bemerkenswerte Einfachheit des Plans (,the obvious crudity of the scheme“, „the remarkable simplicity of the plan") im Vergleich zur Vielzahl, Vielfalt und Unterschiedlichkeit (,diversity“) der Briefe, die der Computer produziert [21, S. 27].

It is clear that these letters are produced by a rather simple trick and that the computer is not really "thinking" at all. This is true of all programs which make the computer appear to think; ... However, sometimes these tricks can lead to quite unexpected and interesting results [21, S. 27].

Aus literaturwissenschaftlicher Perspektive sind Stracheys Experimente komplex und seine Überlegungen dazu aufschlussreich. Er erstellt einen Algorithmus (,scheme“, 
„plan“, „trick“), der eine Maschine dazu bringt, menschliches Denken (im konkreten Fall kann man auch sagen: Dichten) zu imitieren. Erstaunlich dabei ist, dass er das dafür verwendete Wortmaterial nicht einfach aus seiner eigenen Spracherfahrung schöpft, sondern dafür auf eine andernorts gegebene Menge von Wörtern zurückgreift. Im Falle Stracheys ist es Rogets Thesaurus. Bei anderen können Johann Wolfgang Goethe oder Franz Kafka als Quellen dienen, Shakespeare oder Dante, später auch Texte aus Suchmaschinen oder Chats aus sozialen Medien. Was die Auswahl des sprachlichen Materials betrifft, liegen hier avancierte kreative Plagiate vor. Nicht die eigene Sprache wird verwendet, sondern die Sprache anderer, die neu kombiniert und gesampelt wird. Solche Strategien werden später bei vielen anderen Vertreterinnen und Vertretern digitaler Poesie zu finden sein. Auffallend ist Stracheys Beobachtung, dass die Anwendung dieser Strategien zu unerwarteten Ergebnissen führen kann. Genau dies ist Erwartung und Anspruch auch der modernen Digital Humanities, die mit informatischen, statistischen und quantitativen Methoden Texte lesen, analysieren, vergleichen und interpretieren. Eine PHP-Implementierung von Stracheys Programm zur Generierung von Liebesbriefen entwickelte Matt Sephton alias gingerbeardman 2010 im Rahmen einer Ausstellung des Manchester Science and Industry Museum [20].

Stracheys Texte sind leicht als Liebesgrüße oder Liebesbriefe identifizierbar. Nun sind Briefe, insbesondere Liebesbriefe, eine persönliche, bisweilen intime Textsorte. Paradoxerweise lässt Strachey den programmgesteuerten Computer, den er dezidiert als nichtdenkende und infolgedessen nichtmenschliche und nicht der Emotionen fähige Maschine charakterisiert, just diejenige Textsorte hervorbringen, die in ihrer typischen Emotionsintensität das schiere Gegenteil davon zum Ausdruck bringt.

Auffallend ist die Urheberschaftszuschreibung, die Strachey stereotyp in allen Briefen vornimmt. Die Initialen M.U.C. stehen für Manchester University Computer. Der Computer also unterschreibt gewissermaßen den Brief, wodurch ihm die Rolle eines Urhebers oder Autors zugeschrieben wird. Im Eingang seines Essays dagegen hält Strachey fest, dass der programmierende Mensch ursächlich und unverzichtbar sei für das, was der Computer ausführt. Indem er spielerisch den Computer zum Autor deklariert, tritt er als Akteur des Experiments und Autor des Programms in den Hintergrund. Strachey trifft hier eine künstlerische Entscheidung, die zu seinen Erläuterungen über den programmierenden Menschen in spannungsvollem Widerspruch steht. Mit seinen generierten Texten imitiert er menschliches Handeln, Dichten und Denken und emotionalen Ausdruck. Die Imitation menschlicher Kommunikation und das Erschaffen literarischer Texte sind kennzeichnend

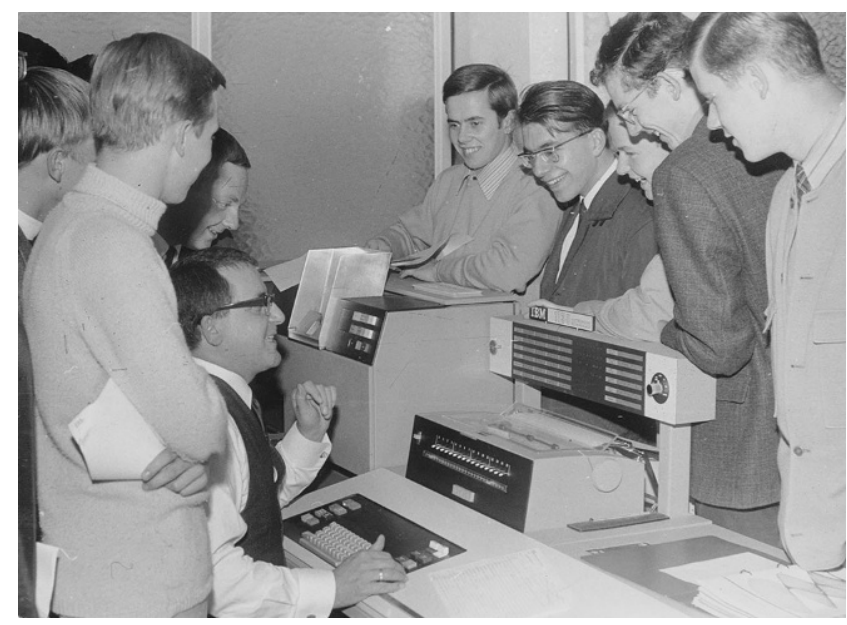

Abb. 1 Theo Lutz (am Bedienpult) mit Studenten bei IBM. Deutsches Literaturarchiv Marbach

für Computerpoesie bis in die Gegenwart und bereits in Stracheys frühen Experimenten deutlich erkennbar.

Was ihren literarischen Charakter betrifft, lassen Stracheys Texte Sinn und Witz erkennen. Sinn stellen sie dadurch her, dass sie einer natürlichsprachigen Syntax folgen und dass die Satzteile grammatikalisch korrekt aneinandergereiht sind. Witz erzeugen sie dadurch, dass sie (scheinbar) mit den Mitteln der Übertreibung und mit den Bedeutungen einzelner Begriffe und der kommunikativen Situation des Briefeschreibens spielen.

Heinz Zemanek (1920-2014), der Wiener Computerpionier und Erbauer des ersten volltransistorisierten Computers auf dem europäischen Festland mit dem Namen „Mailüfterl“, würdigte Strachey als ,eine[n] der geistreichsten Computerpioniere der Welt“. Er hob dessen ,,ausgefallenes Programm ... zum automatischen Schreiben von Liebesbriefen“ hervor und beschrieb es als „eine Anwendung kombinatorischer Flexibilität, nicht ohne lächelnden Seitenblick auf die Redundanz des typischen Liebesbriefs“" [23].

\section{Theo Lutz}

Der im Vergleich zu Strachey 16 Jahre jüngere Theo Lutz (1932-2010) (Abb. 1) begann 1953 sein Studium der Mathematik an der Technischen Hochschule (TH), heute Universität Stuttgart. Sein Ingenieursdiplom erwarb er 1959 am Institut für Theorie der Elektrotechnik mit einer Diplomarbeit über elektrotechnische Netzwerke mit konstanter Dämpfung, insbesondere über Allpässe. Nach seinem Studium arbeitete er als Entwickler für den Elektrogerätehersteller Standard Elektrik Lorenz (SEL) in Stuttgart. 1966 wechselte er zu IBM und war 21 Jahre lang für den Konzern tätig, vorwiegend in Stuttgart, und zwar vor allem 
in der betriebsinternen Wissensvermittlung und Ausbildung sowie als Wissenschaftsjournalist für konzerneigene Journale. Mit der Dissertation Ein statistisches Datenbankmodell (1976) wurde Lutz bei Rul Gunzenhäuser (1933-2018), von dem noch die Rede sein wird, an der Universität Stuttgart im Fach Informatik promoviert. Schon frühzeitig, nämlich 1987, ließ sich Lutz verrenten, war fortan freiberuflicher IT-Berater und Autor von Fach- und Sachbüchern und wurde 1997 zum Honorarprofessor an der Fachhochschule Esslingen berufen. Im Alter befasste er sich intensiv mit mathematikgeschichtlichen Themen, insbesondere mit dem Werk des Renaissancegelehrten, Mathematikers und Reformators Michael Stifel (1487-1567). Sein Nachlass liegt seit 2019 im Archiv des Deutschen Literaturarchivs (DLA) in Marbach am Neckar [5, 6].

\section{Stochastische Texte}

In erster Linie sah sich Lutz als Mathematiker, aber er konnte auch unterhaltsam schreiben. Als Student schrieb er Beiträge für die Theaterbriefe des Kreisjugendrings Esslingen, die eine deutlich antifaschistische Haltung erkennen lassen. Von 1958-1966 war er Chefredakteur der monatlich erscheinenden Jugendzeitschrift ja und nein, die die Entwicklung der noch jungen Bundesrepublik kritisch - und mit $\mathrm{Hu}$ mor - kommentierte. Im Laufe seines Lebens veröffentlichte Lutz zahlreiche Fachbeiträge, Sach- und Fachbücher, darunter Was denkt sich ein Elektronengehirn? (1963, zusammen mit Rolf Lohberg), die Programmierfibel (1965, zusammen mit Volker Hauff) und das Taschenlexikon der Kybernetik (1972). Von seinen wissenschaftsjournalistischen Beiträgen oft zitiert werden seine Prognosen zur Entwicklung der Computertechnologie mit dem Titel Die Zukunft des Computers, die 1984 im Auftrag von IBM erschienen [16, 18]. In Lutz' Nachlass befindet sich das unveröffentlichte Manuskript einer Monographie über Michael Stifel, die den Titel Kollege Stifel trägt.

Aus literatur- und medienwissenschaftlicher Perspektive gelten die Stochastischen Texte von 1959 als Lutz' Hauptwerk $[17,19]$. Es handelt sich dabei um aleatorische Experimente, die Subjekte und Prädikate innerhalb einer festgelegten syntaktischen Struktur nach dem Zufallsprinzip kombinieren. Die Kombination erfolgte aufgrund von sogenannten Pseudozufallszahlen, die mittels Software-Zufallsgenerator gewonnen wurden. Eine Fortsetzung dieser Experimente sind das Weihnachtsgedicht ,und kein engel ist schön" (1960) und ein Liebesgedicht ohne Titel aus dem Jahr $1961[9,15]$. Bis in die 1990er-Jahre experimentierte Lutz weiterhin mit Computerpoesie und nutzte dafür die jeweils neuesten Programmiersprachen und Hardwarelösungen. Sammlungen dieser Versuche, denen Texte von Johann Wolfgang Goethe, Friedrich Schiller, Heinrich Hei- ne, Friedrich Nietzsche, Rainer Maria Rilke, Kurt Schwitters, Martin Heidegger u. a. zugrunde liegen, tragen die Titel „Stochastogramme“, „Stochastolyrik“, „Stochastische Humoresken“, „Geschönte Gedichte“ und „Zufallstexte“. Ein spätes großes Vorhaben trägt den Titel „Aleatoren-Projekt“. Alle diese Arbeiten befinden sich in Lutz' Nachlass, sind allerdings bis heute weitestgehend unbekannt und unerforscht [5, S. 185-186].

Idee und Entstehung der Stochastischen Texte stehen in engem Zusammenhang mit der Diplomarbeit im Fach Elektrotechnik, an der Lutz im Sommersemester 1959 an der TH Stuttgart schrieb. Zu dieser Zeit besaß die Hochschule in ihrem neu gegründeten Recheninstitut 3 Großrechenanlagen: eine Z 22 der Zuse KG aus Bad Hersfeld, eine ER 56 der Standard Elektrik Lorenz (SEL) aus Stuttgart und eine Pegasus von Ferranti aus Manchester. Die Mittel für diese Maschinen kamen aus einem Bundesprogramm zur Förderung der Rechentechnik an deutschen Hochschulen. Doch die teuren Maschinen wurden kaum genutzt [13, S. 121]. Wilhelm Bader (1900-1984), Inhaber des Lehrstuhls für Theoretische Elektrotechnik an der TH Stuttgart, der Lutz' Diplomarbeit betreute, empfahl seinem Schüler, für die Diplomarbeit die vorhandenen Computer zu verwenden. Lutz arbeitete sich gründlich in die $\mathrm{Z} 22$ ein und war bald in der Lage, die Maschine zu programmieren.

Möglicherweise wirkten die Vorlesungen über statistische Ästhetik bei Max Bense (1910-1990), dem einflussreichen Stuttgarter Theoretiker der rationalen Moderne, in Lutz nach, wie bestimmt auch angeregte Diskussionen mit dem Esslinger Schul- und Studienfreund Rul Gunzenhäuser, der zu dieser Zeit ebenfalls Student der Mathematik und Hilfskraft bei Bense war. Im Laufe des Frühjahrs 1959 jedenfalls kam Lutz auf die Idee, mithilfe der Z 22 und unter Verwendung der Subjekt-Prädikat-Struktur einfacher Deklarativsätze sogenannte künstliche Texte, im Besonderen Gedichte, herzustellen. Er benötigte dazu ein Programm sowie Listen mit Wörtern. Begeistert machte er sich an die Arbeit. Die Wörter, die er für das aleatorische Sampling verwenden wollte, extrahierte er aus Gedichten von Goethe. Warum Lutz ausgerechnet Texte Goethes für seine Experimente verwendete, lässt sich nicht abschließend klären. Vermutlich lag die Erwägung zugrunde, dass der als in hohem Maße kanonisch geltende Dichter eine reizvolle Referenz darstellen würde. Von den Versuchen mit Gedichten Goethes sind Arbeitsblätter überliefert, doch ist nicht bekannt, ob Lutz in dieser frühen Phase die Generierung von Texten bereits gelang [5, S. 196].

Vermittelt durch Gunzenhäuser, erfuhr Bense von Lutz' Experimenten. Bense war begeistert, dass dem jungen Studenten nun praktisch gelang, was Bense erst theoretisch modelliert hatte: die Herstellung maschinengemachter Poesie. Am 21. Juli 1959 schrieb Gunzenhäuser Lutz einen Brief. Darin bittet er ihn im Auftrag Benses um die Herstellung 


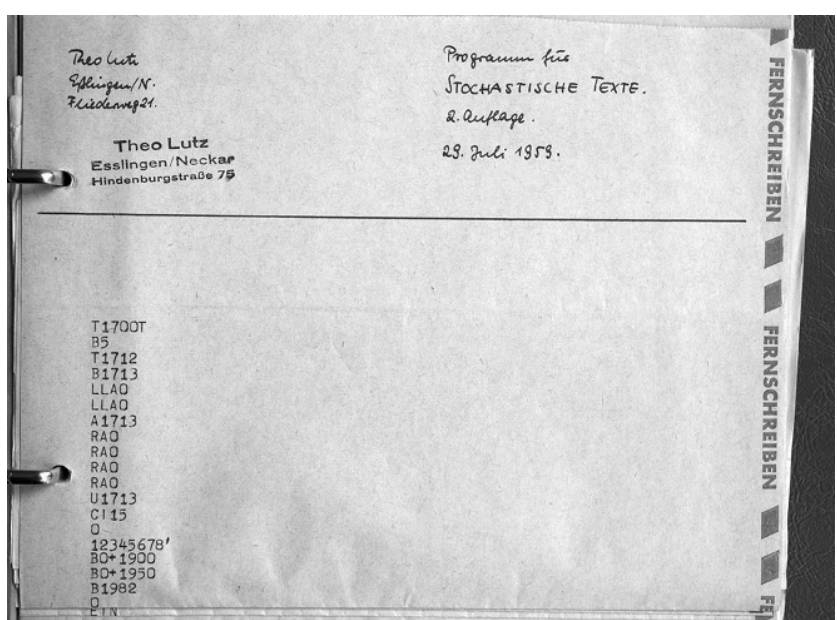

Abb. 2 Die ersten Zeilen des Programms von Theo Lutz zur Erzeugung der Stochastischen Texte. Deutsches Literaturarchiv Marbach

„rein stochastisch erzeugte Texte“, die als Vergleichstexte für Analysen ,allgemeiner Texte“ dienen sollen. Diese Analysen waren Vorarbeiten für Benses Theorie der Texte (1962), an der Bense ab etwa 1958 arbeitete und für die Gunzenhäuser als Hilfskraft engagiert war. Der stochastische Text solle aus sogenannten „Elementarsätzen“ bestehen, die aus 16 Subjekten und 16 Prädikaten gebildet werden. „Elementarsätze“ seien mit einer Wahrscheinlichkeit von 1/8 durch „und“, „oder“ oder „,so gilt" miteinander verbunden, mit einer Wahrscheinlichkeit von 4/8 durch einen Punkt und mit einer Wahrscheinlichkeit von 1/8 durch einen Absatz voneinander getrennt. Subjekte und Prädikate, die technisch bedingt höchstens 10 Schriftzeichen lang sein durften, sollen dem Roman Das Schloss (1926) von Franz Kafka entnommen werden. Diese Entscheidung traf wahrscheinlich Bense, in dessen Verständnis Kafka wohl viel eher der Vorstellung von Moderne entsprach als Goethe, mit dessen Gedichten Lutz experimentierte. Die 16 Subjekte und die 16 Prädikate aus Kafkas Schloss lieferte Gunzenhäuser in seinem Brief gleich mit. Die Verteilung der Subjekte und Prädikate soll gleichwahrscheinlich sein. Lutz machte sich an die Arbeit und kam rasch voran. Innerhalb weniger Tage waren Ende Juli 1959 im Freiburger Code ein lauffähiges Programm (Abb. 2) geschrieben und die stochastischen Texte hergestellt, die Lutz über Gunzenhäuser an Bense schickte [4, S. 329-331, 5, S. 197-198]. Eine PHP-Implementierung von Lutz' Programm legte 2005 der Medienkünstler Johannes Auer vor [2].

Die Abb. 3 zeigt die ersten 6 Zeilen des generierten Texts. Die obere Bildhälfte gibt die Fassung wieder, wie sie aus der Maschine kam, die untere Hälfe den Erstdruck der Veröffentlichung [17, S. 8]. Die Gegenüberstellung macht die Eingriffe sichtbar, die Lutz am Text vornimmt: „AE“ wird zu „, „“ falsch nach einem großen Leerschritt sitzende Punkt wird

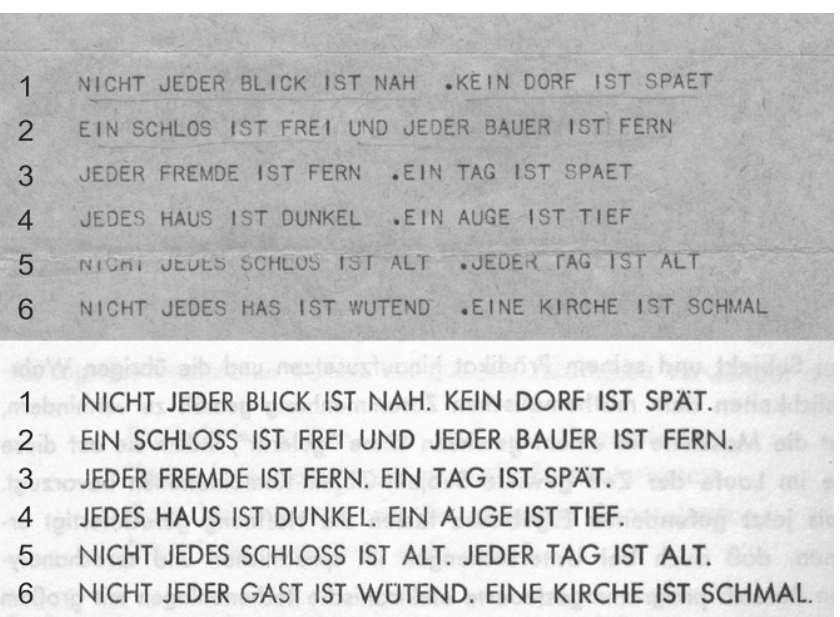

Abb. 3 Die ersten 6 Zeilen der Stochastischen Texte: maschinengenerierter Text (oben) und Erstveröffentlichung (unten) im Vergleich

an die korrekte Stelle gesetzt (Zeilen 1, 3, 4, 5 und 6), am Ende aller Zeilen werden Punkte ergänzt, ebenso fehlendes Doppel-S (Zeilen 2 und 5). Seltsam wirkt im Maschinenausdruck der Satz „NICHT JEDES HAS IST WUTEND“ (Zeile 6). Bei „HAS“ könnte es sich um eine Fehlschreibung (oder Fehlprogrammierung) für „Hase“, „Haus“ oder „Hass“ handeln. Lutz ersetzt „JEDES HAS“ kurzerhand durch „JEDER GAST“: Daran wird deutlich, dass Lutz in seiner Veröffentlichung nicht einfach zeichengleich den maschinengenerierten Text wiedergeben wollte, sondern es für geboten hielt, ihn auch händisch zu korrigieren. Deutlich wird an diesem frühen Beispiel digitaler Poesie darüber hinaus, wie die Mensch-Maschine-Interaktion nicht nur die Programmierung betrifft, sondern entscheidend und gestaltend auch in den Datenoutput eingreift.

Die Sätze sind seriell aneinandergereiht. Der Rhythmus ist stakkatoartig, die Diktion affirmativ. Sinn und Bedeutung des Textes wirken verspielt, dadaistisch, expressiv. Die parataktische Form in tendenziell jambisch drängendem Rhythmus ist auf den vergleichsweise einfachen Algorithmus zurückzuführen, den eine Rechenmaschine im Jahr 1959 bewältigen konnte. Ob es ein öffentliches Echo auf die Publikation gab, ist nicht bekannt. Es folgte aber ein Jahr danach auf einen anderen Text: 1960 generierte Lutz ein Weihnachtsgedicht, das er mit dem Titel ,und kein engel ist schön" versah und unter dem passenden Pseudonym „electronus“ in der Dezember-Nummer von ja und nein veröffentlichte. Die 1. Strophe lautet:

jeder schnee ist kalt und nicht jeder engel ist weiß und nicht jeder schnee ist still und kein friede ist kalt und kein engel ist hell und jeder friede ist still [9] 
Diesmal gelang die Provokation. In der Januar-Nummer 1961 von ja und nein erschienen zahlreiche Zuschriften zum Gedicht. Die Meinungen drifteten auseinander. Sie reichten von Empörung bis Begeisterung [15, S. 3]. Weil die Herstellung von Computerpoesie Lutz offensichtlich großen Spaß bereitete, reichte er in der gleichen Nummer noch ein kurzes Liebesgedicht nach. Er stellt es als „Originalgedicht von ZUSE, in der Handschrift des Dichters“ vor. Die Zuse, gemeint ist die $Z$ 22, die zusammen mit dem Gedicht auch abgebildet wird, umschreibt er als „Elektronengehirn“ und deklariert sie zum „Autor“ [15, S. 3] dieses Gedichts:

kein kuss ist still

oder die liebe ist still

oder keine seele ist rein

und nicht jeder kuss ist gruen

und ein juengling ist heftig. [15, S. 3].

\section{Fazit und Ausblick}

Strachey und Lutz, die beiden Gründerväter digitaler Poesie, arbeiteten form- und traditionsbewusst. Während Strachey die Form des Briefes zerlegt und raffiniert für die Maschine adaptiert, setzt Lutz provokant auf die Zergliederung und Neukombination kanonischer Literatur mit dem Computer. Mag für Strachey das Vorbild englischer Briefromane eine Rolle gespielt haben, so maß sich Lutz an Kafkas „Schloss“ und den intimen Formen romantischer Lyrik. Die Art und Weise der maschinellen Textkombination musste dabei zweierlei berücksichtigen: die Textstruktur und die jeweiligen Codes, die vonnöten waren, um die ersten Computer dazu zu bringen, tatsächlich möglichst wenig stereotype Texte zu produzieren.

Von diesen frühen Texten spannen sich mehrere Entwicklungslinien in die Gegenwart: zum einen die Linie hin zur Netzliteratur, zum anderen diejenige zum literaturaffinen Computerspiel, also etwa zum Storytelling-Experiment oder zu den literaturaffinen Spielen, die Kafka oder Karl Mays Winnetou zum Gegenstand haben. Zugleich zeigt sich, dass auch Enzensbergers berühmter Poesieautomat in der Tradition der frühen digitalen Poesie zu verorten ist: Enzensberger war gut mit Max Bense bekannt und auch mit seinem Interesse an maschineller Dichtung vertraut [1]. Schon im März 1967 veröffentlichte der neugierige Enzensberger darüber ein ganzes Kursbuch mit dem Titel Neue Mathematik. Grundlagenforschung. Theorie der Automaten - ein entscheidender Impuls, der ihn zu seinem Grundlagentext Einladung zu einem Poesie-Automaten (1974) veranlasste. Dort bezieht er sich kursorisch auf die „bislang bekannten Möglichkeiten der programmierten Poesie" und fordert, enthusiastisch linkes Denken, Poesie und maschinellen Fortschritt verbindend: „Poesie-Programmierer aller Länder, vereinigt euch!“ $\left[10\right.$, S. 60, S. 64]. ${ }^{1}$

Wenn es die neue Generation der natürlichsprachigen Algorithmen (NLG) erlaubt, so komplexe Texte zu schaffen, die keine exakte Identifikation der Autorschaft mehr zu erlauben scheinen [14], so ruhen diese auf einer jahrhundertealten Tradition kombinatorischer Literatur und einer beinahe siebzig Jahre alten Tradition digitaler Poesie. Zwar waren die technischen Möglichkeiten in den 1950er-Jahren begrenzt, aber Strachey und Lutz erzeugten doch Verblüffendes, nämlich Texte, die nicht als bloßes Freizeitvergnügen von Informatikern abgetan werden können, sondern Literaturgeschichte schrieben.

Funding Open Access funding enabled and organized by Projekt DEAL.

Open Access Dieser Artikel wird unter der Creative Commons Namensnennung 4.0 International Lizenz veröffentlicht, welche die Nutzung, Vervielfältigung, Bearbeitung, Verbreitung und Wiedergabe in jeglichem Medium und Format erlaubt, sofern Sie den/die ursprünglichen Autor(en) und die Quelle ordnungsgemäß nennen, einen Link zur Creative Commons Lizenz beifügen und angeben, ob Änderungen vorgenommen wurden.

Die in diesem Artikel enthaltenen Bilder und sonstiges Drittmaterial unterliegen ebenfalls der genannten Creative Commons Lizenz, sofern sich aus der Abbildungslegende nichts anderes ergibt. Sofern das betreffende Material nicht unter der genannten Creative Commons Lizenz steht und die betreffende Handlung nicht nach gesetzlichen Vorschriften erlaubt ist, ist für die oben aufgeführten Weiterverwendungen des Materials die Einwilligung des jeweiligen Rechteinhabers einzuholen.

Weitere Details zur Lizenz entnehmen Sie bitte der Lizenzinformation auf http://creativecommons.org/licenses/by/4.0/deed.de.

\section{Literatur}

1. Amslinger T (2018) Verlagsautorschaft: Enzensberger und Suhrkamp. Wallstein, Göttingen

2. Auer J (2005) Theo Lutz (23.07.1932-31.01.2010): Stochastische Texte (1959). https://auer.netzliteratur.net/0_lutz/lutz_original. html. Zugegriffen: 31. Okt. 2020

3. News BBC (2016) Listening to the music of Turing's computer. https://www.bbc.com/news/magazine-37507707. Zugegriffen: 1 . Nov. 2020

4. Bernhart T (2019) Rul Gunzenhäuser und die Stuttgarter Schule der mathematischen Literaturwissenschaften. In: Albrecht A, Bonitz M, Skowronski A (Hrsg) Max Bense: Werk - Kontext - Wirkung. Metzler, Stuttgart, S 323-335. https://doi.org/10.1007/978-3476-04753-3_13

5. Bernhart T (2020) Beiwerk als Werk: „Stochastische Texte“ von Theo Lutz. Editio 34:182-206. https://doi.org/10.1515/editio2020-0010

6. Bernhart T, Richter S (2019) Maschinen können Gedichte schreiben. Süddeutsche Zeitung 244 (22.10.2019): 12. https://www. sueddeutsche.de/digital/literatur-marbach-computer-dichtungpoesie-theo-lutz-1.4649251. Zugegriffen: 31. Okt. 2020

\footnotetext{
${ }^{1}$ Wir danken Xiaocui Qiu für den Hinweis auf die Verbindung von Bense und Enzensberger, die maschinelle Poesie betreffend.
} 
7. Campbell-Kelly M (1985) Christopher Strachey, 1916-1975: A Biographical Note. Ann Hist Comput 7:19-42. https://doi.org/10. 1109/MAHC.1985.10001

8. Copeland J, Long J (2016) Restoring the first recording of computer music. https://blogs.bl.uk/sound-and-vision/2016/09/restoringthe-first-recording-of-computer-music.html. Zugegriffen: 1. Nov. 2020

9. electronus [Pseudonym für Theo Lutz] (1960) und kein engel ist schön. ja und nein. Unabhängige Z Jungen Gener 3(12):3

10. Enzensberger HM (2000) Einladung zu einem Poesie-Automaten. Suhrkamp, Frankfurt am Main

11. Gaboury J (2013) A queer history of computing: part three. https:// rhizome.org/editorial/2013/apr/9/queer-history-computing-partthree/. Zugegriffen: 30. Okt. 2020

12. Harsdörffer GP (1651) Delitiae Mathematicae et Physicae: Der Mathematischen und Philosophischen Erquickstunden Zweyter Theil. Bümler, Nürnberg. http://data.onb.ac.at/rep/10968EDF. Zugegriffen: 1. Nov. 2020

13. Hoffmann C (2004) Eine Maschine und ihr Betrieb: Zur Gründung des Recheninstituts der Technischen Hochschule Stuttgart (1956-1964). In: Büscher B, von Herrmann H-C, Hoffmann C (Hrsg) Ästhetik als Programm: Max Bense / Daten und Streuungen. Vice Versa, Berlin, S 118-129

14. Köbis N, Mossink LD (2021) Artificial intelligence versus Maya Angelou: experimental evidence that people cannot differentiate AI-generated from human-written poetry. Comput Human Behav 114:106553. https://doi.org/10.1016/j.chb.2020.106553
15. Lohberg R (1961) Ehret eure deutschen Dichter. ja und nein. Unabhängige Z Jungen Gener 4(1):2-3

16. Lutz T (o. J.) Die Zukunft des Computers. IBM Österreich. (O. V.), (o. O.)

17. Lutz T (1959) Stochastische Texte. Augenblick Z Tend Exp 4(1):3-9

18. Lutz T (1984) Die Zukunft des Computers. Technik und Gesellschaft 6. IBM Deutschland. (O. V.), (o. O.)

19. Lutz T (2004) Stochastische Texte. In: Büscher B, von Herrmann H-C, Hoffmann C (Hrsg) Ästhetik als Programm: Max Bense / Daten und Streuungen. Vice Versa, Berlin, S 164-169

20. Sephton M (2010) Christopher Strachey „Loveletters“ (1952). https://www.gingerbeardman.com/loveletter/. Zugegriffen: 30. Okt. 2020

21. Strachey C (1954) The „thinking“ machine. Encounter: a monthly review of literature, the arts, and politics: 25-31. http://www.unz. com/print/Encounter-1954oct-00025/. Zugegriffen: 26. Okt. 2020

22. Suter B (2012) Von Theo Lutz zur Netzliteratur: Die Entwicklung der deutschsprachigen elektronischen Literatur. In: Auer J, Heibach C, Suter B (Hrsg) netzliteratur.net: Netzliteratur / Internetliteratur / Netzkunst (http://docplayer.org/2116205-Von-theolutz-zur-netzliteratur-die-entwicklung-der-deutschsprachigenelektronischen-literatur-von-beat-suter.html. Zugegriffen: 31. Okt. 2020)

23. Zemanek H (1975) Nachruf für Christopher Strachey. Inf Technol 17(4):159. https://doi.org/10.1524/itit.1975.17.16.159. Zugegriffen: 27. Okt. 2020 